\title{
Comparison of Curvilinear Stiffeners and Tow Steered Composites for Aeroelastic Tailoring of Transports
}

\author{
Bret K. Stanford* \\ NASA Langley Research Center, Hampton, VA, 23681 \\ Christine V. Jutte ${ }^{\dagger}$ \\ Craig Technologies, Inc., Cape Canaveral, FL, 32920
}

\begin{abstract}
A series of aeroelastic optimization problems are solved on a high aspect ratio wingbox of the Common Research Model, in an effort to minimize structural mass under coupled stress, buckling, and flutter constraints. Two technologies are of particular interest: tow steered composite laminate skins and curvilinear stiffeners. Both methods are found to afford feasible reductions in mass over their non-curvilinear structural counterparts, through both distinct and shared mechanisms for passively controlling aeroelastic performance. Some degree of diminishing returns are seen when curvilinear stiffeners and curvilinear fiber tow paths are used simultaneously.
\end{abstract}

\section{Introduction}

The design optimization of flexible wingbox structures via composite laminates affords the optimizer a large design space (relative to metallic components) with which to tune the aeroelastic behavior. The stacking sequence details of each skin panel may be tailored for the desired in-plane properties ( $\boldsymbol{A}$ constitutive matrix) and bending properties ( $\boldsymbol{D}$ constitutive matrix). The $\boldsymbol{A}$ matrix of each panel, when assembled into a wingbox structure, govern the global deformation properties of the wing, such as maneuver load response or flutter. The $\boldsymbol{D}$ matrix of each panel governs the local deformation of that panel, namely the buckling response. Various couplings known to be important for aeroelastic response, such as bend-twist coupling or shearextension coupling, may be tailored into the structure through various stacking sequence designs. An early well-known paper by Shirk et al. ${ }^{1}$ details many of these themes.

There has been recent interest in further expanding the composite design space for wingbox structures through the use of tow steering. Tow steered composites are created with automated fiber placement machines, which can lay fibers along precise curvilinear paths to create variable-stiffness panels. ${ }^{2}$ Each layer of a laminate may be steered independently, or the paths of each layer may be linked in order to preserve potentially-desirable laminate features such as balance or symmetry. For a wingbox composed of rib-delineated skin panels, a single steering path may be utilized from root to tip, with plies added or deleted from one panel to the next. Aeroelastic tailoring via tow steered composites is demonstrated in Refs. $3-6$, as are benefits to localized skin buckling performance, ${ }^{7}$ and load paths around cutouts. ${ }^{8}$

A second tailoring scheme of interest in this work is curvilinear skin stiffeners: curved metallic subcomponents constructed with additive manufacturing. ${ }^{9}$ If metal skins are utilized, the entire stiffened panel may potentially be built as a single piece through the metal deposition process. If, as in the case of this paper, composite skins are used, the metallic curvilinear stiffeners are fastened to the panels. A third possibility is the use of composite stiffeners, but this is not utilized here.

The benefits of using curvilinear stiffeners for panel optimization, driven by buckling and stress metrics, have been demonstrated in Refs. 10 and 11. Expanding this framework to an entire wingbox has proven to be a challenge, however, due to the high computational cost of capturing and tracking all of the local buckling modes in between the various curved stiffeners in each panel of the wing, as well as numerous global

* Research Aerospace Engineer, Aeroelasticity Branch, bret.k.stanford@nasa.gov, AIAA Senior Member.

${ }^{\dagger}$ Research Engineer, Advanced Materials and Processing Branch, christine.v.jutte@nasa.gov. 
modes. A simpler industry-standard approach is taken here, by smearing the curved stiffeners into the skin panel $^{12}$ for the purposes of computing stiffness properties. These properties (namely the shell's $\boldsymbol{A}, \boldsymbol{B}$, and $\boldsymbol{D}$ matrices, where $\boldsymbol{B}$ represents coupling between in-plane and out-of-plane mechanics) will then spatially vary along the length of the panel in much the same way as for a tow steered composite, to account for the spatial variation in stiffener direction and pitch (spacing).

The goals of this work are to demonstrate aeroelastic optimization of a high aspect ratio Common Research Model wingbox using curvilinear stiffeners and/or tow steered composites. The mass of the wing structure is minimized subject to aeroelastic stress, buckling, and flutter constraints, spread across several trimmed maneuver loads. A series of optimization problems are solved with increasingly-complex structural parameterizations:

1. A metallic wingbox with straight stiffeners.

2. A metallic wingbox with curved stiffeners.

3. A composite wingbox with straight stiffeners and straight fibers.

4. A composite wingbox with straight stiffeners and curved fibers.

5. A composite wingbox with curved stiffeners and straight fibers.

6. A composite wingbox with curved stiffeners and curved fibers.

Comparisons between designs 1 and 2 will quantify metallic weight reductions available through curvilinear stiffener design variables. Design 3 similarly serves as a composite baseline for the curvilinear designs 4, 5, and 6. Design 6 is of particular interest, as it will be indicative of any synergistic relationships between tow steered fibers and curvilinear stiffeners, in terms of both the best-available weight reduction and the spatial distribution of stiffness/load.

\section{Common Research Model}

All of the work in this paper is conducted on a conceptual high aspect ratio Common Research Model (CRM). The $1 g$ outer mold line for a lower aspect ratio (9) CRM is described in Ref. 13, and a jig shape CRM wingbox subsequently developed by Kenway et al. ${ }^{14}$ A span extension of this model increased the aspect ratio from 9 to 13.5; the latter configuration (shown in Fig. 1) is used here. This transonic transport has a wing span of $72 \mathrm{~m}$, a mean aerodynamic chord of $6.3 \mathrm{~m}$, a taper ratio of 0.25 , a sweep angle of $35^{\circ}$, and a cruise Mach number of 0.85 .

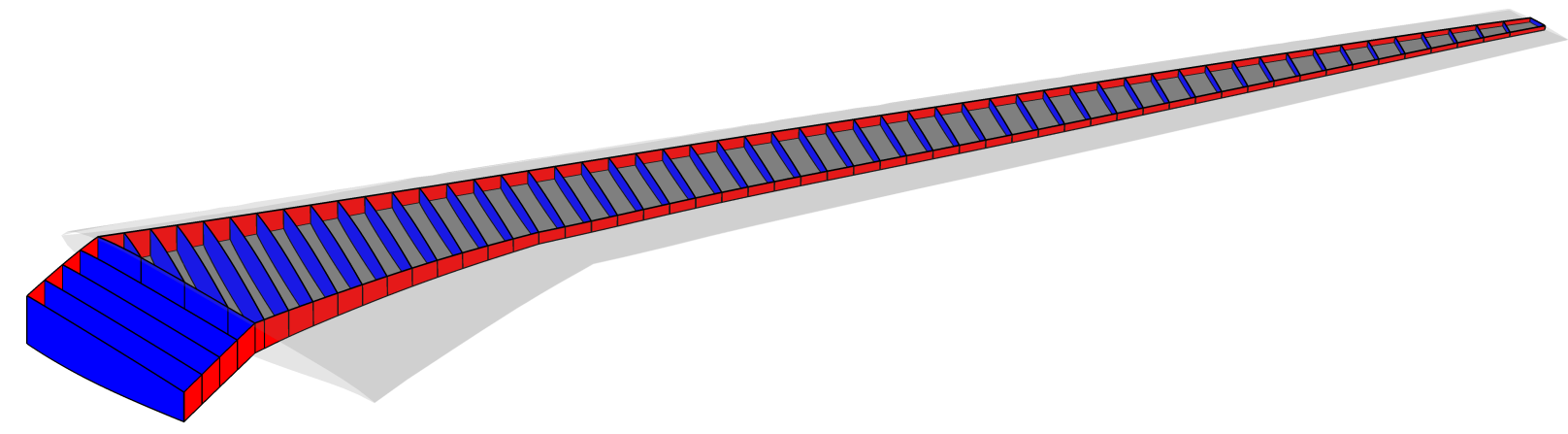

Figure 1. Wingbox and outer mold line of the high aspect ratio CRM.

The topology of the wingbox in Fig. 1 consists of 58 ribs, leading and trailing spars, and upper and lower surface skins. The leading spar is straight, and spans between $10 \%$ chord at the root and $35 \%$ at the tip. The trailing spar has a slight break at $31 \%$ of the semi-span (where the planform does as well), and spans between $60 \%$ chord at the root, $70 \%$ at this break location, and $60 \%$ at the tip. All shell members are outfitted with T-shaped stiffeners, where the flange is bonded to the shell members. The thickness of the flange and the web are equal for all cases, as is the width of the flange and the height of the web. The stiffeners are not modeled explicitly, but instead smeared into the shell stiffness properties. ${ }^{12}$ The stiffener pitch is equal to $15.1 \mathrm{~cm}$ for the skins, $17.6 \mathrm{~cm}$ for the spars, and $19.9 \mathrm{~cm}$ for the ribs. For the skins, parallel run-out stiffeners are utilized down the span. In the absence of curvilinearity, all skin stiffeners are parallel to the leading spar: curvature design variables will alter this axis, and the local pitch as well. 
The wing structure (carry-through plus main wing) is discretized in 21,000 triangular shell finite elements. All nodes along the centerline are given symmetric boundary conditions, and all nodes along the wing root (side-of-body) are pinned. Though not shown in Fig. 1, lumped mass representations (attached to the wingbox with interpolation elements) are used to model control surfaces $(4,400 \mathrm{~kg})$, an engine $(7,400 \mathrm{~kg})$, and fuel $(45,000 \mathrm{~kg}$ for full fuel). Non-modeled mass (fuselage, payload, etc.) for the half-vehicle is fixed at $75,000 \mathrm{~kg}$. As will be seen below, typical structural mass values for the wingbox range from 13,000 to 16,000 $\mathrm{kg}$, so the TOGW for the entire vehicle is roughly $290,000 \mathrm{~kg}$.

Aerodynamic paneling for the wing, horizontal tail, vertical tail, fuselage, and engine (the latter two represented as cruciforms) is shown in Fig. 2, with a total of 5,000 panels. For static aeroelastic trim analysis, the entire vehicle representation of Fig. 2 is utilized. For dynamic flutter analysis, only the wing panels are utilized.

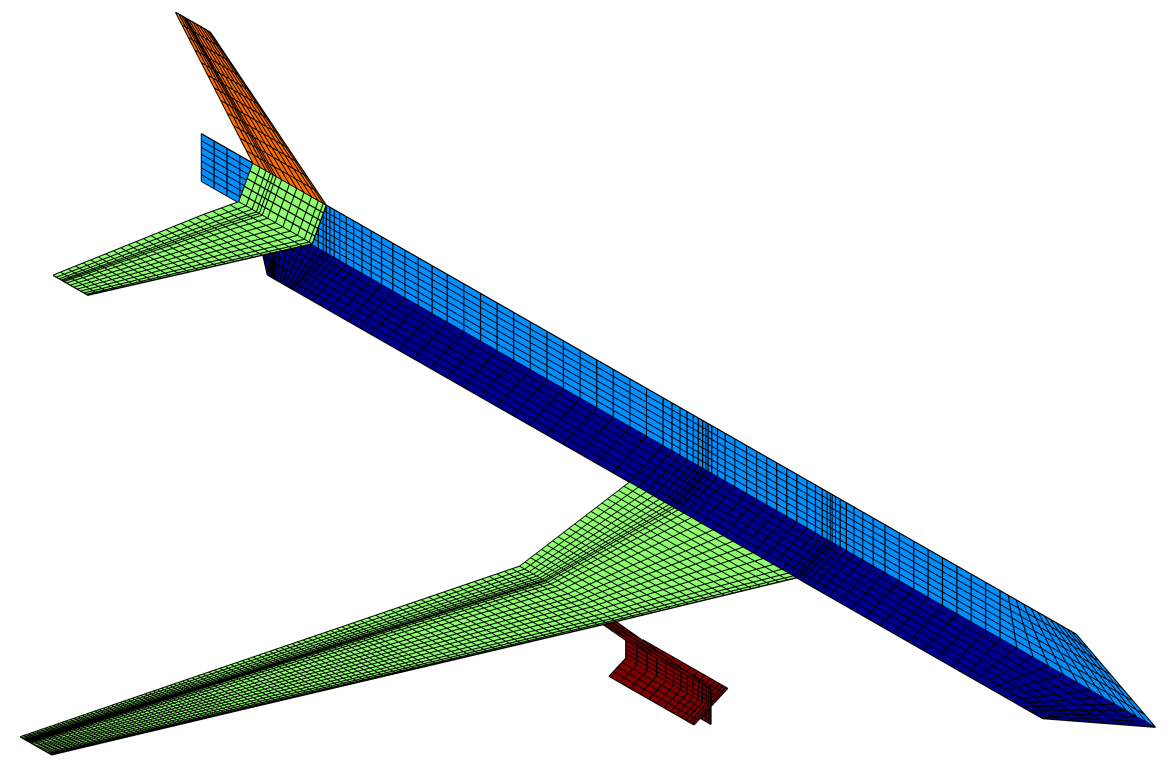

Figure 2. Aerodynamic paneling of the high aspect ratio CRM.

\section{Aeroelastic Modeling and Sensitivities}

\section{A. Static Aeroelastic Maneuvers}

The shell finite elements used to model the wing structure are defined by a combination of linear strain triangles (LST) and discrete Kirchhoff triangles (DKT). ${ }^{15}$ For static airloads, a linear vortex lattice method ${ }^{16}$ is used to model the aerodynamic lifting surfaces. A finite plate spline (FPS) method ${ }^{17}$ is used to transfer downwash and pressures between the aerodynamic and structural modules. Only information pertaining to the wing is transferred in this way: the remaining aerodynamic surfaces are not explicitly tied to any structure.

The wingbox structure is sized across three different types of static maneuvers. The first type is a longitudinal maneuver (pull up, push over), where the system is trimmed via the angle of attack, $\alpha$, and the elevator deflection, $\delta$ :

$$
\left[\begin{array}{cccc}
\boldsymbol{K} & -q \cdot \boldsymbol{Q} & \mathbf{0} & \mathbf{0} \\
-\boldsymbol{P} & \boldsymbol{D}_{s} & -\boldsymbol{L}_{\alpha} & -\boldsymbol{L}_{\delta} \\
\mathbf{0} & q \cdot \boldsymbol{S}_{L}^{T} & 0 & 0 \\
\mathbf{0} & q \cdot \boldsymbol{S}_{m}^{T} & 0 & 0
\end{array}\right] \cdot\left\{\begin{array}{c}
\boldsymbol{u} \\
\boldsymbol{C}_{p} \\
\alpha \\
\delta
\end{array}\right\}=\left\{\begin{array}{c}
N \cdot \boldsymbol{F}_{\text {grav }}+\boldsymbol{F}_{\text {thrust }} \\
\boldsymbol{L}_{j i g} \\
N \cdot W \\
0
\end{array}\right\}
$$

The first row of Eq. 1 is the finite element analysis: $\boldsymbol{K}$ is the stiffness matrix, and $\boldsymbol{u}$ is the solution vector. Forcing functions include self-weight inertial loading, $\boldsymbol{F}_{\text {grav }}$ (scaled by the load factor $N$ ), thrust loading, $\boldsymbol{F}_{\text {thrust }}$, from the engine, and aerodynamic forces. Aerodynamic forces are written as $q \cdot \boldsymbol{Q} \cdot \boldsymbol{C}_{p}$, where $\boldsymbol{C}_{p}$ is 
a vector of differential pressure coefficients acting on each panel, $Q$ is an interpolation function derived from FPS, and $q$ is the dynamic pressure.

The second row of Eq. 1 is the aerodynamic analysis, where $\boldsymbol{D}_{s}$ is the matrix of aerodynamic influence coefficients (AIC) and the subscript indicates a symmetric aerodynamic condition about the centerline of the airplane in Fig. 2. This equation is driven by downwash due to angle of attack, $\boldsymbol{L}_{\alpha} \cdot \alpha$ (where $\boldsymbol{L}_{\alpha}$ is a linear operator that converts the scalar angle of attack into a downwash at each panel), elevator deflection, $\boldsymbol{L}_{\delta} \cdot \delta$, built in camber/twist of the wing and tail jig shapes, $\boldsymbol{L}_{j i g}$, and downwash induced by structural wing deformation. This latter term is written as $\boldsymbol{P} \cdot \boldsymbol{u}$, where $\boldsymbol{P}$ is a second interpolation function, also derived from FPS-based methods.

Trim equations are written in the 3rd and 4th rows of Eq. 1: $q \cdot \boldsymbol{S}_{L}$ and $q \cdot \boldsymbol{S}_{m}$ convert the differential pressure vector, $\boldsymbol{C}_{p}$, into a total aerodynamic lift and aerodynamic pitching moment (about the aircraft center of gravity). Lift must offset the total weight of the vehicle $(N \cdot W)$, and the pitching moment must be zero.

A second type of static maneuver considered here is a rolling trim analysis Eq. 2, where the deflection, $\beta$, of an outboard wing aileron is found such that a constant specified non-dimensional roll rate, $p \cdot L / U$, is maintained, with no rolling acceleration. In this analysis, $p$ is the dimensional roll rate, $L$ is the semi-span, $U$ is the flight speed, and the aileron is placed between $70 \%$ and $90 \%$ of the semi-span, with a hinge line at $71 \%$ of the local chord. The system is simultaneously trimmed longitudinally for steady level flight $(N=1)$ with the angle of attack, $\alpha$. The rolling analysis requires an anti-symmetric condition about the centerline of the airplane; the longitudinal analysis uses a symmetric condition:

$$
\left[\begin{array}{cccccc}
\boldsymbol{K} & \mathbf{0} & -q \cdot \boldsymbol{Q} & \mathbf{0} & \mathbf{0} & \mathbf{0} \\
\mathbf{0} & \boldsymbol{K} & \mathbf{0} & -q \cdot \boldsymbol{Q} & \mathbf{0} & \mathbf{0} \\
-\boldsymbol{P} & \mathbf{0} & \boldsymbol{D}_{s} & \mathbf{0} & -\boldsymbol{L}_{\alpha} & \mathbf{0} \\
\mathbf{0} & -\boldsymbol{P} & \mathbf{0} & \boldsymbol{D}_{a} & \mathbf{0} & -\boldsymbol{L}_{\beta} \\
\mathbf{0} & \mathbf{0} & q \cdot \boldsymbol{S}_{L}^{T} & \mathbf{0} & 0 & 0 \\
\mathbf{0} & \mathbf{0} & \mathbf{0} & q \cdot \boldsymbol{S}_{p}^{T} & 0 & 0
\end{array}\right] \cdot\left\{\begin{array}{c}
\boldsymbol{u}_{s} \\
\boldsymbol{u}_{a} \\
\boldsymbol{C}_{p, s} \\
\boldsymbol{C}_{p, a} \\
\alpha \\
\beta
\end{array}\right\}=\left\{\begin{array}{c}
N \cdot \boldsymbol{F}_{\text {grav }}+\boldsymbol{F}_{\text {thrust }} \\
\mathbf{0} \\
\boldsymbol{L}_{j i g} \\
(p \cdot L / U) \cdot \boldsymbol{L}_{p} \\
N \cdot W \\
0
\end{array}\right\}
$$

The $s$ and $a$ subscripts indicate "symmetric" or "anti-symmetric" terms, $q \cdot \boldsymbol{S}_{p}$ converts the aerodynamic pressures into a rolling moment about the centerline (which is set to zero for a constant roll rate), and $\boldsymbol{L}_{p}$ converts the rolling motion into a downwash term.

The final type of load case considered here is an inertial-only load case, with neither aeroelasticity, trimming mechanisms, nor control surface actuation involved. This is meant to emulate landing loads or taxi bump loads, with wing deformation simply computed with Eq. 3.

$$
\boldsymbol{K} \cdot \boldsymbol{u}=N \cdot \boldsymbol{F}_{\text {grav }}
$$

A final summary of the four load cases used for this work is given in Table 1.

Table 1. Summary of static aeroelastic load cases.

\begin{tabular}{rrrrrr} 
Case & $N$ & $p \cdot L / U$ & Mach & Altitude & Equation \\
\hline 1 & 2.5 & - & 0.85 & $10,000 \mathrm{ft}$ & 1 \\
2 & -1 & - & 0.6 & $0 \mathrm{ft}$ & 1 \\
3 & 1 & 0.03 & 0.85 & $30,000 \mathrm{ft}$ & 2 \\
4 & 3 & - & - & - & 3
\end{tabular}

\section{B. Stresses}

Having solved for the wing deformation in Eqs. 1, 2, or 3, stresses and strains can be computed for each static load case, and a knock-up (safety) factor is applied to each elemental stress value: 1.5 is used for metallic components, 1.7 for composites. Stress-based failure functions are computed for each finite element: the von Mises failure function is used for metallic elements, the Tsai-Wu failure criteria for composites. The wingbox is then divided into patches, where each skin panel (delineated by adjacent ribs) is a patch, each rib is a patch, and each spar segment seen in Fig. 1 is a patch, for 283 total patches. The Kreisselmeier-Steinhauser (KS) function ${ }^{18}$ is used to compress all of the elemental failure function values within a set of adjacent 
patches into a single metric. If the KS function for a given patch set is less than one, all of the stress tensors within each of the associated patches are within their failure envelope.

\section{Panel Buckling}

After the stress analysis, buckling analyses are run for each stiffened panel in the upper and lower surface skins. Following Ref. 19, each buckling analysis is conducted with a Rayleigh-Ritz method (assumed buckling modes). A typical panel buckling analysis ${ }^{12}$ would consider both global buckling (using smeared material properties, with simply supported boundary conditions along the two rib and two spar segment attachment lines) and local inter-stiffener buckling (using un-smeared material properties and simply supported boundary conditions along the two rib and two stiffener attachment lines). However, a key focus of this work is the use of curvilinear stiffeners, which would create inter-stiffener panels with curved boundaries. Non-rectangular panels are not amenable to the Rayleigh-Ritz method being used here, and so the local inter-stiffener buckling metrics are neglected for this work; only global panel buckling is considered.

The buckling equation is given as:

$$
\left(\boldsymbol{K}_{s}+\mu_{n} \cdot \tilde{\boldsymbol{K}}\right) \cdot \boldsymbol{v}_{n}=\mathbf{0}
$$

where $\boldsymbol{v}_{n}$ is the eigenvector (modal amplitude) associated with the $n^{\text {th }}$ eigenvalue, $\mu_{n}$. The panel stiffness matrix, $\tilde{\boldsymbol{K}}$, is based upon the smeared stiffness properties defined at the wing level. The panel's geometric stiffness matrix, $\boldsymbol{K}_{s}$, is assembled with element stresses computed from Eqs. 1, 2, or 3. An eigenvalue, $\mu_{n}$, greater than one is unacceptable, and a value greater than the applied safety factor (as above, 1.5 and 1.7 for metallics and composites, respectively) is indicative of a buckled state. Three modes $(n=3)$ are computed for each panel buckling eigenproblem, and then eigenvalues are grouped with adjacent panel values to form a smaller number of KS metrics. As above, if the KS value for a given set of panels is less than one, each of those panels is within its buckling failure envelope.

\section{Flutter}

Flutter analysis is conducted using only the wing aerodynamic paneling in Fig. 2. A dynamic eigenvalue problem is written as: ${ }^{20}$

$$
\left(\left(U^{2} / b^{2}\right) \cdot p_{n}^{2} \cdot \overline{\boldsymbol{M}}+(U / b) \cdot p_{n} \cdot \overline{\boldsymbol{C}}+\overline{\boldsymbol{K}}-q \cdot \boldsymbol{A}(i \cdot k)\right) \cdot \boldsymbol{u}_{n}=\mathbf{0}
$$

where $b$ is half the mean aerodynamic chord, $\boldsymbol{u}_{n}$ is the eigenvector associated with the $n^{\text {th }}$ complex eigenvalue $p_{n}, k$ is the reduced frequency, $\overline{\boldsymbol{M}}$ is the mass matrix, $\overline{\boldsymbol{C}}$ is the damping matrix, and $\overline{\boldsymbol{K}}$ is the stiffness matrix. The overbar signifies a reduced term, where the natural vibration mode shapes of the wing have been used to compress full order terms down to modal quantities. The generalized aerodynamic forces, $\boldsymbol{A}$, are written in the frequency domain and are computed as follows. For a fixed wing shape, the steady nonlinear background flow is computed with the Euler code ZEUS ${ }^{21}$ for a range of Mach numbers. For each flow condition, ZTRAN ${ }^{22}$ is used to compute oscillatory perturbations about this mean flow (via the timelinearized transonic small disturbance equations), and then output aerodynamic influence coefficients (AICs) for a range of reduced frequencies, $k$. These AICs are converted into $\boldsymbol{A}$ via the FPS-based interpolation functions $\boldsymbol{P}$ and $\boldsymbol{Q}$ from above, as well as the vibration mode shapes.

For a given Mach number, Eq. 5 is solved across a range of matched-point equivalent airspeeds using a non-iterative $\mathrm{p}-\mathrm{k}$ method. The method of Ringertz ${ }^{23}$ is used to formulate a flutter constraint. If each eigenvalue is written as $p=g+i \cdot k$, then for a given Mach number, the damping $(g)$ of each mode is constrained to lie below a given curve at all equivalent airspeeds, $U_{E A S}$, of interest:

$$
g \leq \begin{cases}0 & 0 \leq U_{E A S} \leq U^{*} \\ c \cdot\left(U_{E A S}-U^{*}\right)^{2} & U_{E A S}>U^{*}\end{cases}
$$

where $c$ is a scaling parameter, and $U^{*}$ is the minimum allowable flutter equivalent airspeed, based on a $15 \%$ margin. Critical $U_{E A S}$ points (local minima) of the inequality in Eq. 6 are computed and lumped together into a single KS constraint. As above, if this constraint is greater than one, then Eq. 6 is not satisfied, and the structure does not meet the required flutter margin. A separate KS constraint is utilized for each considered Mach number. 


\section{E. Analytical Sensitivities}

Derivatives of the static aeroelastic response (stress and buckling aggregation parameters for each load case) with respect to all design variables, are computed with an adjoint method. This method is advantageous as the number of constraints is consistently less than the number of design variables, for a given load case. ${ }^{24}$ Sensitivities of the flutter response are computed via nonlinear eigenvalue derivative methods. ${ }^{25}$ The nonlinearity here refers not to the transonic effects (the method is entirely linearized with regards to this), but to the fact that $\boldsymbol{A}(i \cdot k)$ is a complex function of the eigenvalue $p_{n}$. A free-mode dynamic derivative approach is utilized here, where the derivative of the mode shapes with respect to design variables is neglected for the purposes of gradient computations. Mode shapes are actually updated from one design iteration to the next, however.

\section{Metallic Wing Designs}

\section{A. Straight Stiffeners}

First considering an all-aluminum wingbox $\left(E=73 \mathrm{GPa}, \nu=0.33, \rho=2780 \mathrm{~kg} / \mathrm{m}^{3}, \sigma_{Y}=420 \mathrm{MPa}\right)$, the structure is broken into 283 patches (one per skin panel, one per rib, and one per spar segment seen in Fig. 1). The thickness of each patch, the thickness of the stiffeners attached to each patch, and the height of these stiffeners are all optimized, resulting in 849 total design variables. Skin stiffeners are, for now, fixed as straight and parallel to the leading edge spar throughout. Wing mass (based on the volume of the finite element model) is minimized subject to a variety of constraints:

$$
\min \text { mass s.t. } \begin{cases}\mathbf{0} \leq \boldsymbol{q} \leq \mathbf{1} & \\ K S_{\sigma, i} \leq 1 & i=1, \ldots,\left(N_{\sigma} \cdot N_{L}\right) \\ K S_{\mu, i} \leq 1 & i=1, \ldots,\left(N_{\mu} \cdot N_{L}\right) \\ K S_{f, i} \leq 1 & i=1, \ldots, N_{\text {mach }} \\ K S_{t / t_{s}} \leq 1 & \\ K S_{A R} \leq 1 & \end{cases}
$$

where $\boldsymbol{q}$ are the design variables that have been normalized between 0 and 1 . Dimensionally, shell thicknesses are allowed to range between $3 \mathrm{~mm}$ and $30 \mathrm{~mm}$, stiffener thicknesses between $2.5 \mathrm{~mm}$ and $30 \mathrm{~mm}$, and stiffener heights between $30 \mathrm{~mm}$ and $150 \mathrm{~mm}$ (except for vertical stiffeners on the ribs and spars, whose lower height bound is set to $64 \mathrm{~mm}$ : this higher value helps prevent local vibration modes in these areas).

In Eq. 7, $N_{L}$ is the number of static load cases in Table $1, K S_{\sigma}$ are the stress aggregation parameters $\left(N_{\sigma}\right.$ per load case), $K S_{\mu}$ are the buckling aggregation parameters $\left(N_{\mu}\right.$ per load case), and $K S_{f}$ are the flutter aggregation parameters (monitored at Mach numbers of $0.75,0.8,0.875$, and 0.8812 ). It is desired that, for a given design patch, the shell thickness be within $2.5 \mathrm{~mm}$ of the stiffener thickness. These constraints are normalized to 1 for each design patch, and then all aggregated into a single metric, $K S_{t / t_{s}}$. Similarly, the aspect ratio of each stiffener should not be greater than 15 , which is compressed into the single constraint, $K S_{A R}$.

Each set of structural design variables (shell thickness, stiffener thickness, and stiffener height) is passed through a linearly-decaying cone-shape filter ${ }^{26}$ in order to prevent the difference in stiffness between adjacent patches from being too large. A linear matrix relationship can be built between the unfiltered design variables (which are directly created by the optimizer), and the filtered design variables (which are used to construct the aeroelastic model). All gradient-based optimization problems are solved with the Globally-Convergent Method of Moving Asymptotes tool (GCMMA). ${ }^{27}$

The resulting optimal structural design variables are shown in Fig. 3 (design 1), in terms of the shell thickness, smeared stiffener thickness, and smeared stiffener height of each design patch. The structural mass of this wing (objective function in Eq. 7) is $16,146 \mathrm{~kg}$. The optimizer places the majority of the material in the upper and lower surface skins, with peak thickness at roughly $30 \%$ of the semi-span (coinciding with the slight break in the trailing spar), and thickness tapered towards the lower bound at the wing tip. Rib design variables are mostly pushed to the lower bounds, which may be due to the omission of crushing loads in the structural analysis used here. Stiffeners are also pushed to their lower bounds in most of the webs (ribs and spars), which again may be due to the omission of shear-web buckling metrics. 
A notable exception to these two trends is toward the wing tip, where the optimizer has allocated a large amount of material in the webs. This is driven by inertial considerations, namely to satisfy the aggressive flutter constraints, $K S_{f}$, by decreasing the bending frequencies: this outboard mass is not seen if the flutter constraint is removed (a result not shown here). The $3 g$ inertial load case in Table 1 is included here partly to counteract this outboard material allocation, but the optimizer is still able to pursue this strategy, and also satisfy the stress and buckling constraints associated with this load case (as will be seen). This compromise may be the reason that the optimizer has not allocated the mass at the extreme wing tip, where it would presumably have the greatest flutter impact.

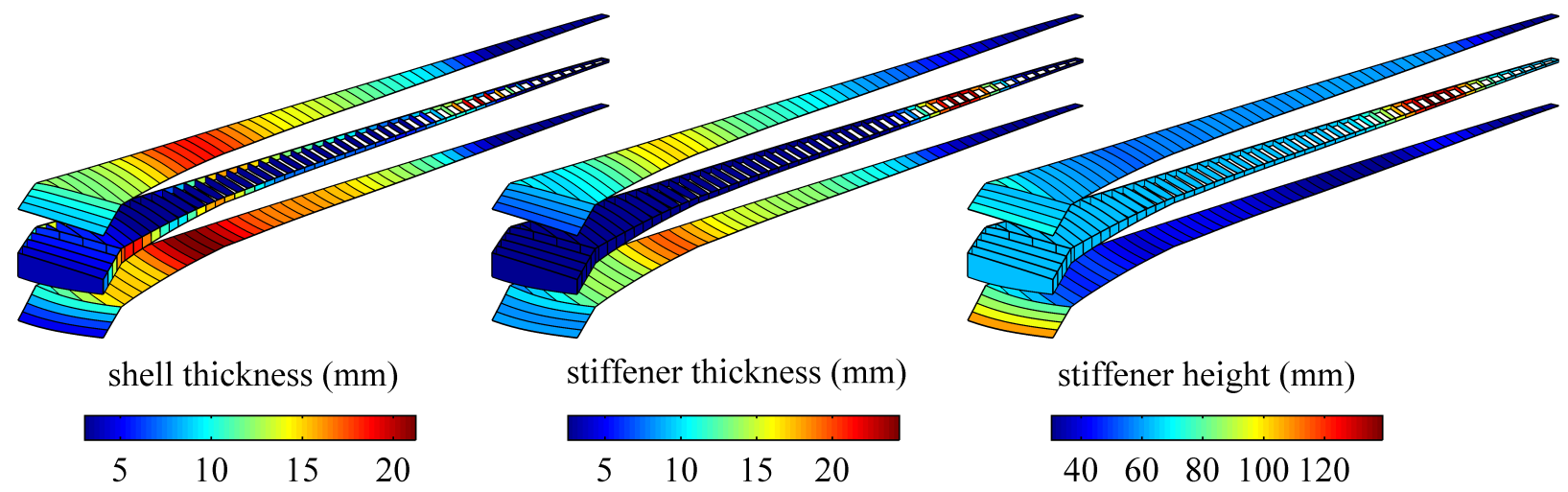

Figure 3. Optimal structural design variables for the metallic wing with straight stiffeners: design 1.

Structural deformation and stress-based failure indices for the four load cases are seen in Fig. 4. Deflections are fairly large for this configuration: $18.5 \%$ and $19.7 \%$ of the semi-span for the $2.5 \mathrm{~g}$ pull up (load case 1 ) and the roll maneuver (case 3), respectively. Several $K S_{\sigma}$ constraints for all cases except load case 2 are active (i.e., equal to 1), but the failure indices in Fig. 4 are slightly less than one, owing to the conservatism of the KS formulation. There is more torsion in case 3 than case 1 (24\% more wash-out tip twist), and this has drawn the area of peak stress further out towards the tip. The inertial load case 4 is stress-critical only at the wing root spar break (an expected stress concentration), in contrast with the aeroelastic load cases, whose peak stresses are spread over a greater portion of the wing. This would suggest that load case 4 is a weaker design driver than cases 1 and 3 .

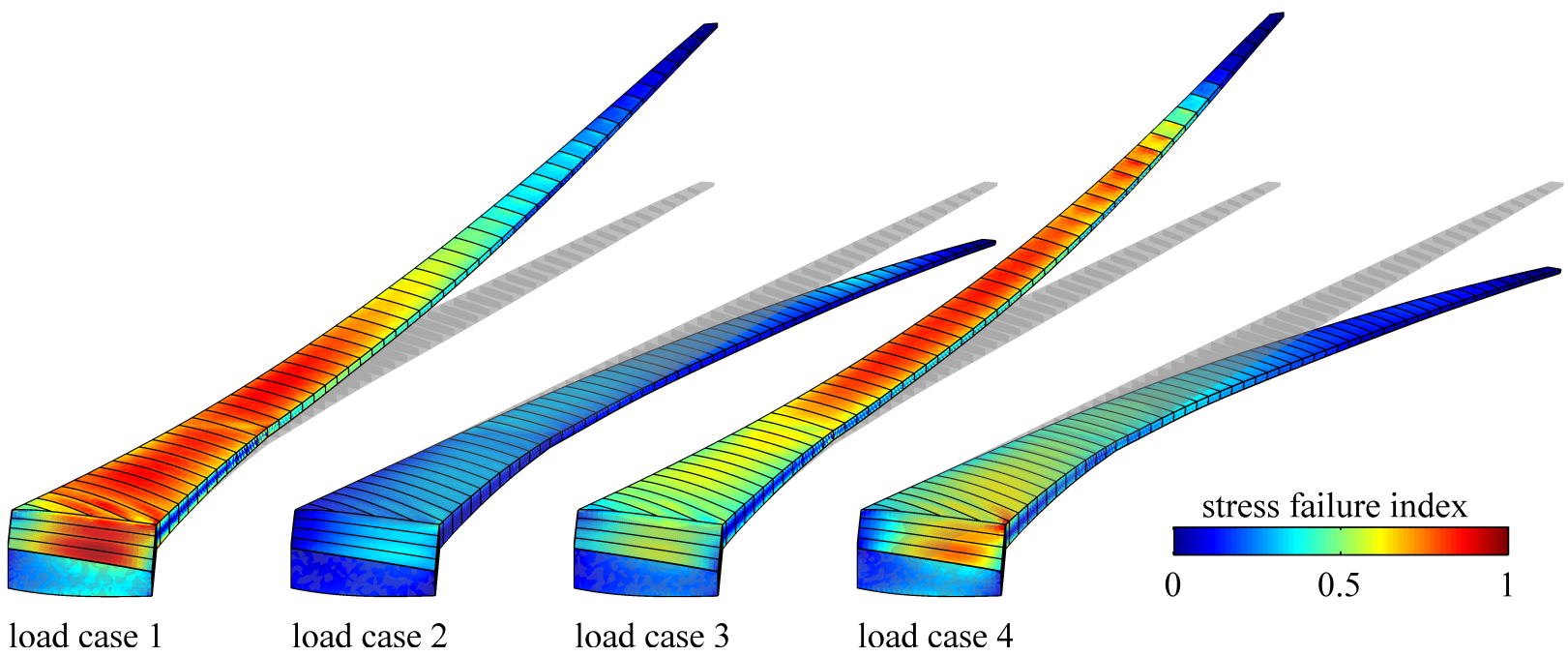

Figure 4. Structural displacements and stress-based failure indices for design 1.

Buckling failure indices are shown in Fig. 5. For the positive load cases, buckling is only active for the compressed panels along the upper surface, and vice-versa for the negative load cases. As with the previous stress results, several $K S_{\mu}$ buckling constraints are active for each load case (even the $-1 g$ push over, whose 
stresses in Fig. 4 are well below their failure boundary), but the panel eigenvalues shown in Fig. 5 are slightly less then one, due to the conservatism of the KS formulation.

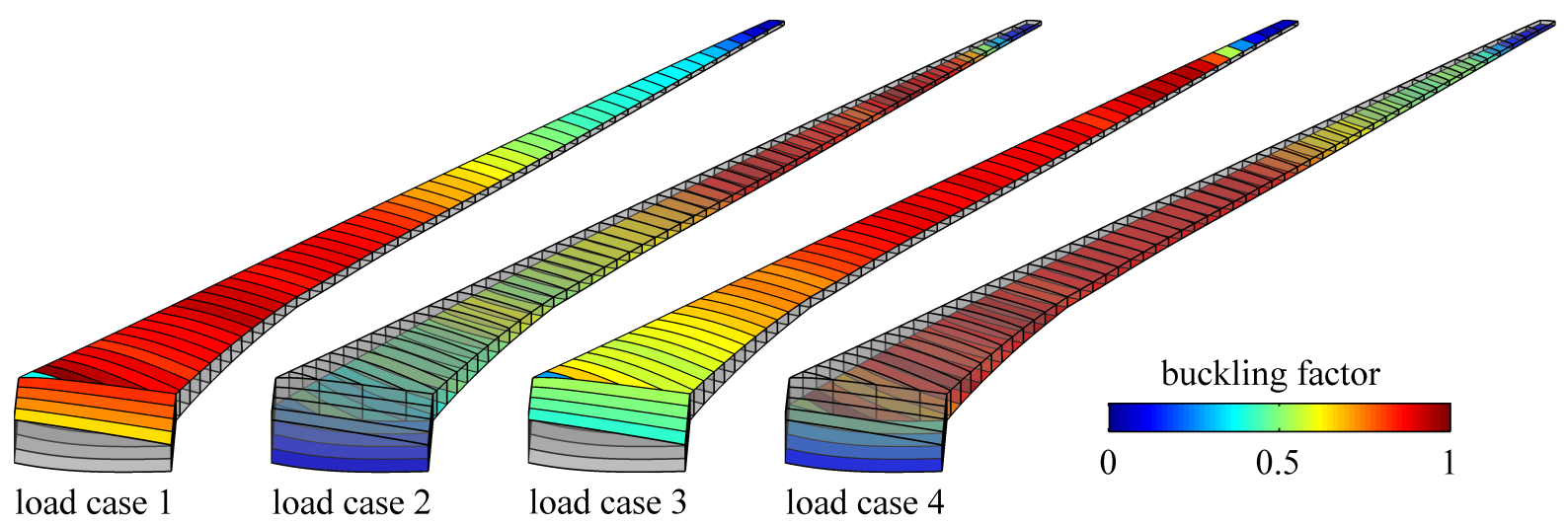

Figure 5. Buckling failure indices for design 1.

Lastly, the flutter behavior for this design is shown in Fig. 6, in terms of the flutter speed, $U_{E A S}$, as a function of Mach number (left), and eigenvalue migration across $U_{E A S}$ for Mach numbers 0.75 and 0.875 . A dive speed of $185 \mathrm{~m} / \mathrm{s}$ is assumed, and $U^{*}$ in Eq. 6 is $15 \%$ beyond this. The flutter constraint is obviously active for Mach numbers 0.75 and 0.875 (a "hard" flutter crossing for the former, a lightly damped hump mode for the latter, as seen in the right plots), but is active at 0.8 as well. This is not evident from the left plot of Fig. 6, as the optimizer has pushed a critical hump mode completely below the constraint boundary in Eq. 6, completely stabilizing it. $K S_{f}$ for this mach number is active, but the system actually flutters at a higher margin than $15 \%$. This quenching of hump modes is the reason for the apparent discontinuous behavior in the left plot of Fig. 6 . Mach 0.875 represents the minimum of the transonic "dip", beyond which flutter speed, $U_{E A S}$, rises sharply with Mach number.
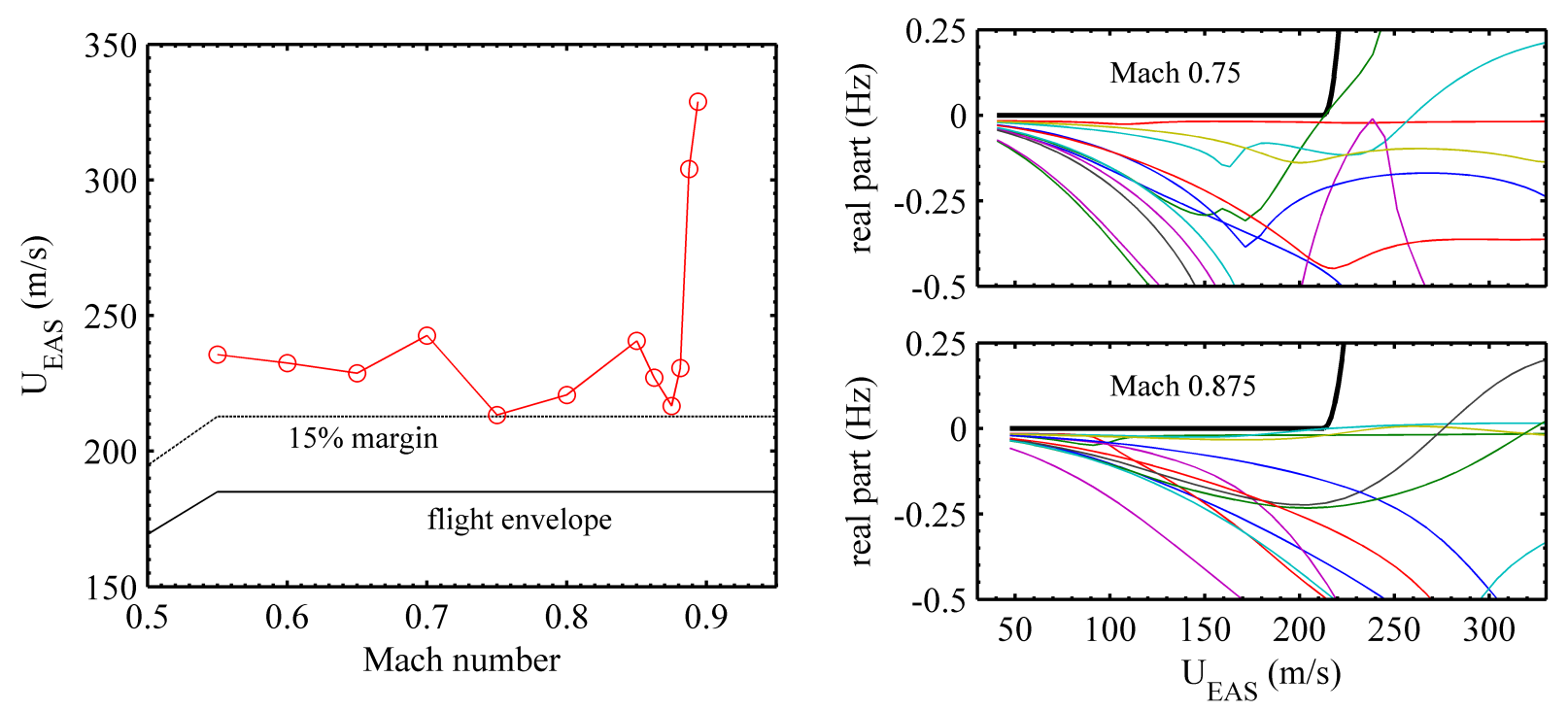

Figure 6. Flutter behavior for design 1: thick black line in plots on right is the constraint boundary of Eq. 6.

\section{B. Curvilinear Stiffeners}

The results in the previous section have utilized straight stiffeners in the skins, parallel to the leading spar throughout. This section still considers an all-metallic wingbox, but augments the optimization problem with curvilinear skin stiffener variables. Stiffeners in the ribs and spars remain straight. The curvilinear path of the stiffeners is parameterized as follows: a control point (knot) is located at the center of each rib, and the optimizer controls the path angle deviation at each knot. If the path deviations are all set to $0^{\circ}$, 
then the stiffeners remain parallel to the leading spar. Positive angles orient the stiffeners ahead of the wing tip as one moves down the span, and angle deviations are bounded (during optimization) between $\pm 70^{\circ}$. The path angle deviation is assumed to vary linearly within each rib bay, ${ }^{28}$ as dictated by the two bounding control knots. The actual stiffener path can be computed by integrating the piecewise-linear function of angle deviations along the wing: an example of this process is given in Fig. 7.

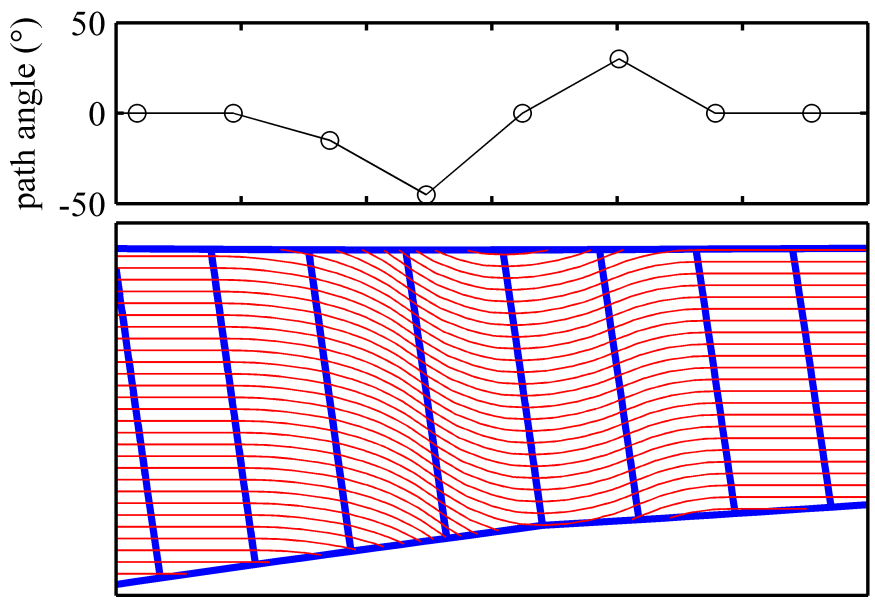

Figure 7. Example set of curvilinear stiffeners (bottom), and corresponding path angle control knots (top).

Curving a stiffener path impacts both the stiffness and mass of the smeared panel. For example, the in-plane constitutive matrix for a given panel is computed as: ${ }^{12}$

$$
\boldsymbol{A}=\frac{E \cdot t}{1-\nu^{2}} \cdot\left[\begin{array}{ccc}
1 & \nu & 0 \\
\nu & 1 & 0 \\
0 & 0 & \frac{1-\nu}{2}
\end{array}\right]+2 \cdot t_{s} \cdot h_{s} \cdot \frac{E}{p_{0} \cdot \sin (\phi)} \boldsymbol{T}^{T}(\phi) \cdot\left[\begin{array}{ccc}
1 & 0 & 0 \\
0 & 0 & 0 \\
0 & 0 & 0
\end{array}\right] \cdot \boldsymbol{T}(\phi)
$$

with similar expressions for the $\boldsymbol{B}$ and $\boldsymbol{D}$ matrices. The first term is the contribution from the isotropic shell with thickness, $t$. The second term is the smeared contribution from the stiffeners with thickness, $t_{s}$, and height, $h_{s}$, multiplied by 2 to account for the T-shape. The stiffener angle is $\phi$, and is used to form the trigonometric tensor transformation matrix, $\boldsymbol{T}$, which orients the effective stiffness axis. The pitch of the straight stiffeners is $p_{0}$ : this term is scaled by $\sin (\phi)$, to account for the spatial compression of the curved/angled stiffeners, clearly seen in Fig. 7.

The mass of the panel changes through two factors. The spatial compression of the curved stiffeners through decreased pitch (reflected by $p_{0} \cdot \sin (\phi)$ in Eq. 8) will increase the mass, as will the increase in the stiffener path's arc length from rib to rib. It is finally noted that, whereas a panel with straight stiffeners has uniform properties throughout (within the confines of the smearing assumption), stiffness and mass properties become spatially-dependent for a curvilinearly-stiffened panel.

The optimization problem of Eq. 7 is repeated here with 108 additional design variables governing the path angle control knots at each rib: a separate set for the upper and lower skins. The shell thickness, stiffener thickness, and stiffener height design variables are still optimized for each design patch, and the optimal design of Fig. 3 (with straight stiffeners) is used as a starting point for the optimizer. The optimal mass for this case (design 2) is $15,308 \mathrm{~kg}$, a $5.2 \%$ reduction relative to the previous result, afforded by the expanded design space.

The equivalent thickness distribution (including the smeared effect of the stiffeners) of the wingbox structures with and without curvilinear stiffeners are compared in Fig. 8, and the optimal stiffener paths through the upper and lower skins are shown in Fig. 9. Material reductions within the inboard skins and spars are obtained by attenuating the stress concentrations, curving the stiffeners around the discontinuities in the wing geometry (especially at the side-of-body, seen in the right plot of Fig. 9). The optimizer also succeeds in largely removing the web mass located toward the tip, identified above as being utilized to satisfy the flutter constraint. Greater control of the load paths via curvilinear stiffeners allows for a stiffness-centric solution to meeting this flutter constraint, as opposed to an inertial-centric solution (and its concomitant weight penalty), by angling the stiffener path ahead of the wing tip. 
A final feature of note in Fig. 9 is the large stiffener curvature towards the wing tip (95\% of the semispan), particularly in the lower skins. High stiffener curvature will increase the panel mass via an increase in path arc length (and decrease in pitch), and so this effect may be partially driven by the flutter constraint as well. However, high curvatures in this area are also noted for optimization cases (not shown here) without a flutter constraint; furthermore, the trend is exacerbated for cases (also not shown) with more aggressive roll maneuvers (e.g., higher trimmed roll rates). It is likely that this feature is a result of the high loading into the wing from the aileron used to drive this roll maneuver, specifically to keep these panels from buckling, given the critical $K S_{\mu}$ values in this area seen in Fig. 5.

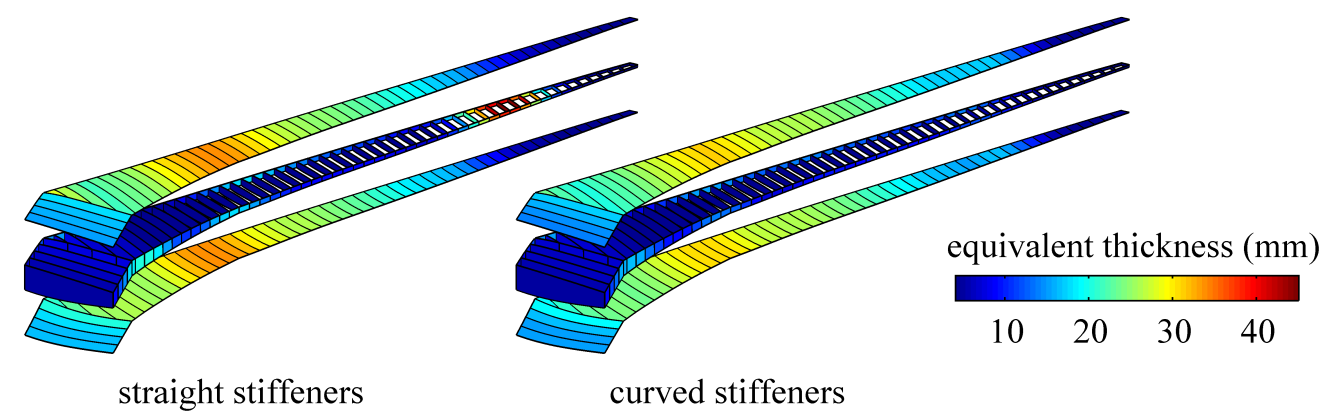

Figure 8. Equivalent thicknesses for the cases with (design 2) and without (design 1) curvilinear stiffeners.
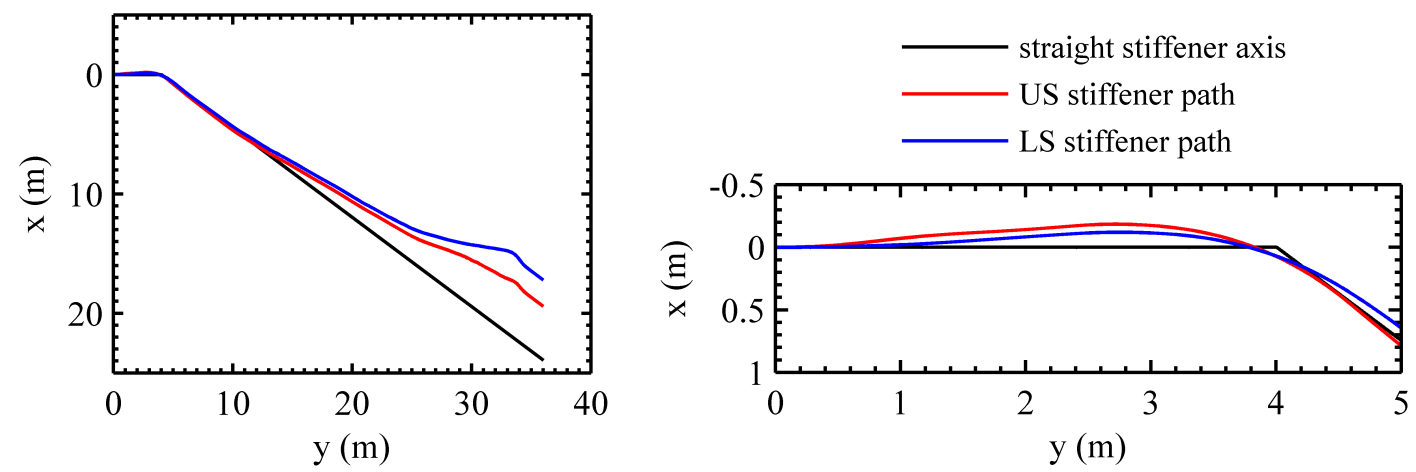

Figure 9. Curved stiffener paths for design 2: right plot is a zoomed-in detail of the left.

\section{Composite Wing Designs}

This section uses composite laminates for the upper and lower wing skins; the ribs, spars, and stiffeners (either straight or curved) are still composed of aluminum. Composite ply material properties are listed in Table 2. In this work, ply angles are restricted to $0^{\circ}, 45^{\circ},-45^{\circ}$, and $90^{\circ}$ (where the $0^{\circ}$ direction is aligned with the wing's leading edge), a typical restriction for aircraft structures rooted in certification concerns. ${ }^{29}$ All laminates are further restricted to be symmetric and balanced (specially orthotropic), and so every $45^{\circ}$ ply must be accompanied by a $-45^{\circ}$ ply.

Table 2. Composite material properties.

\begin{tabular}{rrrrrr} 
Parameter & Value & Parameter & Value & Parameter & Value \\
\hline$E_{1}$ & $128 \mathrm{GPa}$ & $E_{2}$ & $11 \mathrm{GPa}$ & $G_{12}$ & $4.5 \mathrm{GPa}$ \\
$\nu_{12}$ & 0.25 & $\rho_{\text {ply }}$ & $1522 \mathrm{~kg} / \mathrm{m}^{3}$ & $t_{\text {ply }}$ & $0.125 \mathrm{~mm}$ \\
$X_{1 t}$ & $1170 \mathrm{MPa}$ & $X_{1 c}$ & $1120 \mathrm{MPa}$ & $S_{12}$ & $48 \mathrm{MPa}$ \\
$X_{2 t}$ & $40 \mathrm{MPa}$ & $X_{2 c}$ & $170 \mathrm{MPa}$ & &
\end{tabular}

The parameterization scheme of Liu and Haftka ${ }^{30}$ is used here, which combines ply thickness variables and laminate parameter variables for each skin panel. Because $45^{\circ}$ and $-45^{\circ}$ plies must be paired to maintain 
balance, the number of 2-ply stacks is a natural choice to parameterize the ply thickness: $n_{0}$ and $n_{90}$ are the number of 2-ply stacks in the $0^{\circ}$ and $90^{\circ}$ directions, and $n_{45}$ is the number of $\pm 45^{\circ}$ pairs. The total laminate thickness is then $\left(n_{0}+n_{45}+n_{90}\right) \cdot 4 \cdot t_{p l y}$, and the stacking sequence is a permutation of the form $\left[\left(0^{\circ}\right)_{2 \cdot n_{0}} /\left( \pm 45^{\circ}\right)_{n_{45}} /\left(90^{\circ}\right)_{2 \cdot n_{90}}\right]_{s}$.

The four flexural laminate parameters are used to implicitly control this stacking sequence, though one of them $\left(W_{2}\right)$ is small enough to ignore for specially orthotropic laminates, and another $\left(W_{4}\right)$ is exactly zero for symmetric laminates. The constitutive matrices for each laminate are then written as:

$$
\begin{gathered}
\boldsymbol{A}=\left(\left(n_{0}+n_{45}+n_{90}\right) \cdot \boldsymbol{\Gamma}_{0}+\left(n_{0}-n_{90}\right) \cdot \boldsymbol{\Gamma}_{1}+\left(n_{0}-n_{45}+n_{90}\right) \cdot \boldsymbol{\Gamma}_{3}\right) \cdot 4 \cdot t_{p l y}+\boldsymbol{A}_{\text {stiff }} \\
\boldsymbol{D}=\left(\boldsymbol{\Gamma}_{0}+W_{1} \cdot \boldsymbol{\Gamma}_{1}+W_{3} \cdot \boldsymbol{\Gamma}_{3}\right) \cdot\left(\left(n_{0}+n_{45}+n_{90}\right) \cdot 4 \cdot t_{p l y}\right)^{3} / 12+\boldsymbol{D}_{\text {stiff }}
\end{gathered}
$$

$\boldsymbol{\Gamma}_{i}$ are material invariant matrices, ${ }^{19} W_{1}$ and $W_{3}$ are the remaining non-zero laminate parameters, and $\boldsymbol{A}_{\text {stiff }}$ and $\boldsymbol{D}_{\text {stiff }}$ are contributions from the smeared stiffeners (see Eq. 8). The laminate contribution to the $\boldsymbol{B}$ matrix is zero due to the symmetry requirement, but the eccentric stiffeners contribute to this term, as above. Laminate stiffness is then a function of 5 design variables: $n_{0}, n_{45}, n_{90}, W_{1}$, and $W_{3}$. The two laminate parameters may not be chosen entirely independently of the two-ply stack variables, since not all combinations will correspond to an actual laminate. Six constraints, written in terms of the 5 design variables in Ref. 30, must be enforced to ensure feasibility.

The minimum-mass optimization process is similar to that used in the previous section, with composite design variables $n_{0}, n_{45}, n_{90}, W_{1}$, and $W_{3}$ for each of the upper and lower skin panels, metallic thickness patch design variables in the ribs and spars, and stiffener variables (thickness, $t_{s}$, and height, $h_{s}$ ) for every patch. Skin stiffeners are fixed as straight for now. Additional constraints to the problem of Eq. 7 include the 6 laminate feasibility constraints per panel, and a constraint on the minimum allowable ply fraction (10\%) for each fiber direction in each panel. Explicit manufacturing constraints that dictate ply addition and deletion between adjacent panels are not included here, but all design variables are again passed through a spatial filter that will prevent large lay-up differences between adjacent panels. The optimal result (design 3 ) is shown in Fig. 10, with a structural mass of $15,036 \mathrm{~kg}$, a $6.9 \%$ reduction relative to the all-metallic design with straight stiffeners (Fig. 3).
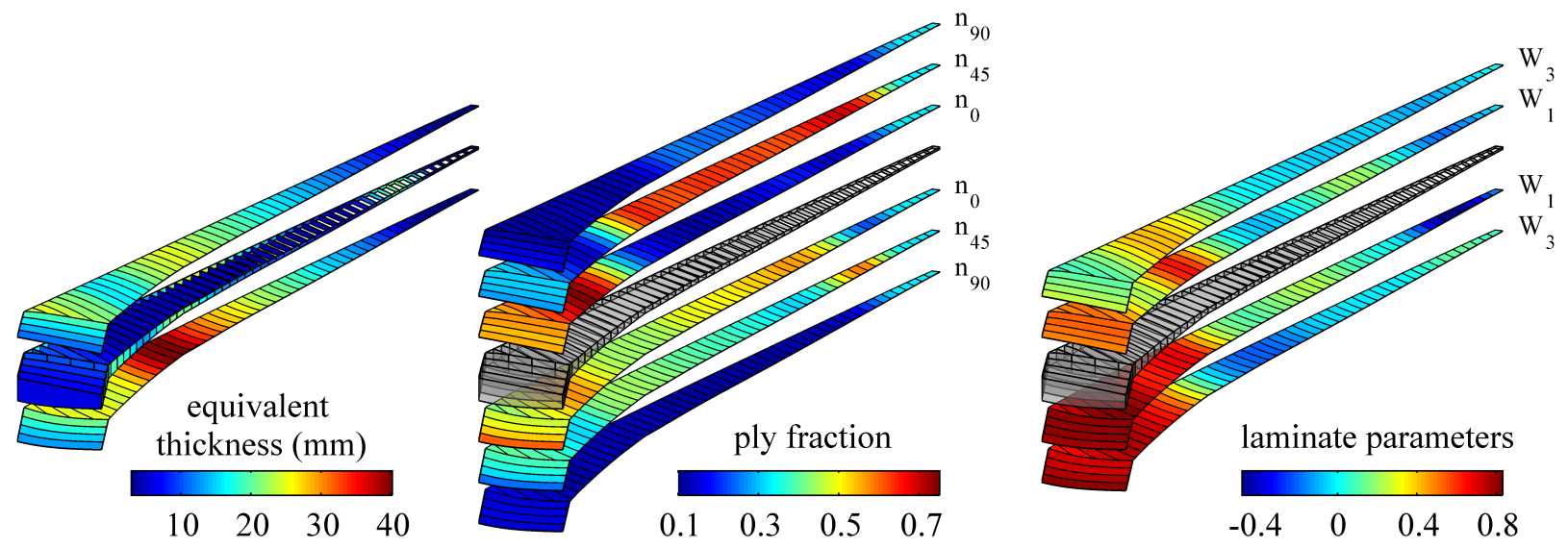

Figure 10. Optimal structural design for the composite wing with straight stiffeners and fibers (design 3 ).

The equivalent thickness on the left of Fig. 10 accounts for both shell thickness (laminates in the skins, metallics in the webs) and smeared stiffener properties, correcting for the difference in densities between the two materials. The optimizer still utilizes inertial web mass towards the tip to help satisfy the flutter constraints, though not as strongly as done for the metallic design (left side of Fig. 8). It is also noted that the equivalent thickness in the upper skins is far less than the lower skins (they are roughly equal for the metallic designs in Fig. 8), and that the upper skins also tend to rely on laminates which are dominated by a single fiber direction: $0^{\circ}$ fibers toward the root and $\pm 45^{\circ}$ outboard. The latter use of $\pm 45^{\circ}$ fibers is driven by the rolling maneuver, which imparts large torsional stresses in this area of the wingbox (Fig. 4). The lower skins utilize laminates composed of a more equal mix of the two fiber directions, though neither 
skin sets utilize many $90^{\circ}$ fibers, whose $10 \%$ minimum allowable ply fraction is an active constraint through much of the wing.

\section{A. Tow Steering}

The result in Fig. 10 utilizes straight fibers (and straight stiffeners as well), with the $0^{\circ}$ direction aligned with the wing leading edge. The composite design parameterization is now augmented to include tow steered fibers. In theory, each ply in a laminate can be steered independently, though the resulting laminate would not, in general, be composed of only $0^{\circ}, 45^{\circ},-45^{\circ}$, and $90^{\circ}$ fibers. This feature (special orthotropy) is to be preserved in order to continue the use of the parameterization scheme implemented above. Specially orthotropic laminates can be utilized in a steering environment by laying all of the $0^{\circ}$ stacks along some optimized steering path, forcing the $90^{\circ}$ stacks along courses perpendicular to this path at each location in the laminate, and the $\pm 45^{\circ}$ stacks along paths deviating by $45^{\circ}$ or $-45^{\circ}$ from the path. Globally, fiber angles can take any value, but locally, each point in a given panel has the same laminate (same number of two-ply stacks, same stacking sequence). ${ }^{3}$

The design of the steering path is accomplished with the same methods used for curvilinear stiffeners in Fig. 7. A path angle control point (knot) is associated with each rib, and the tow steering path angle is linearly interpolated within each rib bay. As before, if the path deviations are all set to $0^{\circ}$, then the $0^{\circ}$ fibers remain parallel to the leading spar. Positive angles orient the fibers ahead of the wing tip, and angle deviations are bounded (during optimization) between $\pm 70^{\circ}$.

The composite optimization problem of the previous section is repeated here with 108 additional design variables parameterizing the tow steered paths, separately for the upper and lower skins. The straight fiber result of Fig. 10 is used as a starting point, and the optimizer is able to exploit the expanded design space by reducing the structural mass down to $13,700 \mathrm{~kg}$, an $8.8 \%$ reduction relative to the straight fiber design. Comparisons between the two designs are given in Fig. 11, in terms of equivalent skin thickness and tow steering patterns (the path of the $0^{\circ}$ fibers), with clear reductions in the former metric when optimized with tow steering.
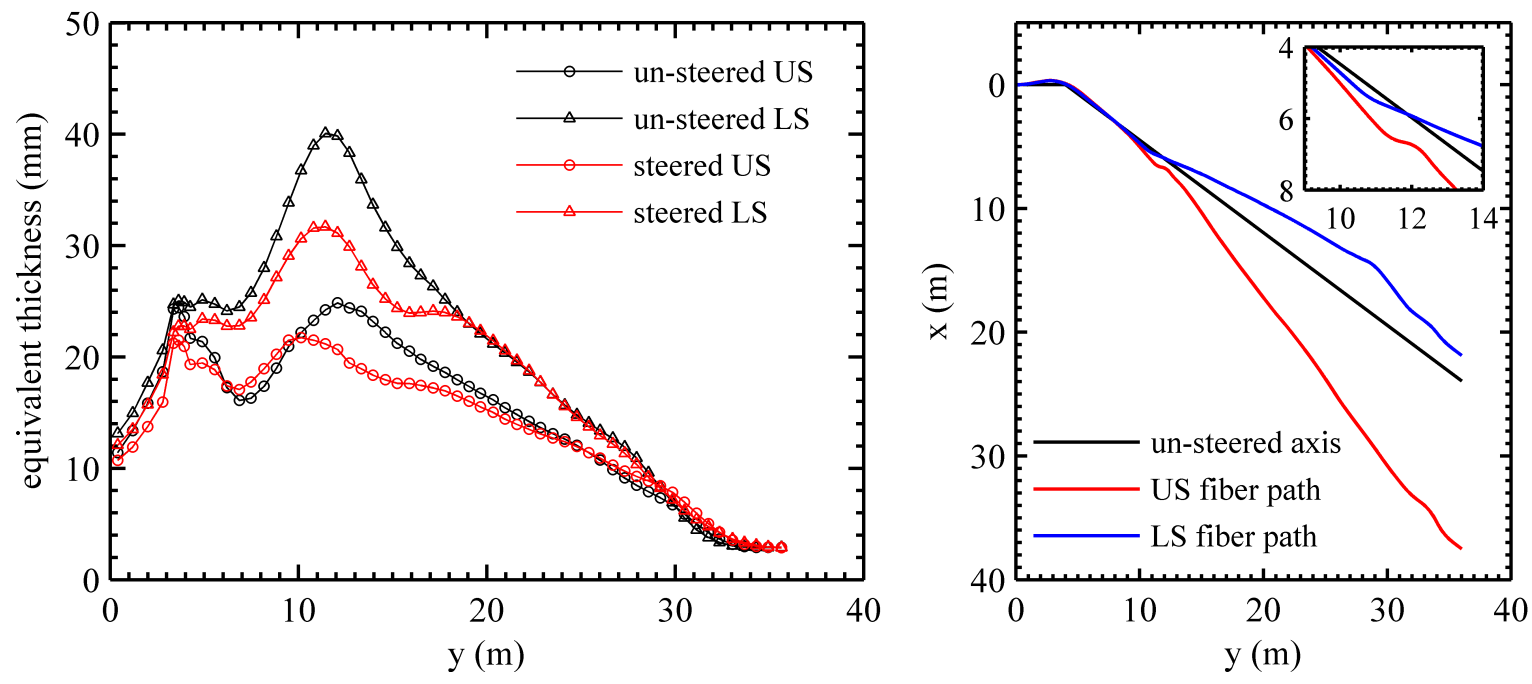

Figure 11. Equivalent skin thicknesses and steering patterns for the optimal tow steered result (design 4 ). Steering patterns for this case are also shown in the top of Fig. 14.

Large portions of the steered $0^{\circ}$ fiber paths in Fig. 11 simply deviate from the un-steered axis (angling ahead of the wing tip along the lower skins, and behind the wing tip in the upper skins) without providing high degrees of curvature within a given panel. This simply gives the optimizer an ability to use ply fiber angles outside the predetermined set of $0^{\circ} / 45^{\circ} /-45^{\circ} / 90^{\circ}$, and is not necessarily a referendum on the aeroelastic benefits of tow steering. Large localized fiber curvature is noted, however, in the vicinity of the outboard aileron and the side-of-body spar break, as also observed in the previous curvilinear stiffener result. The largest curvature is seen in the upper skins at the secondary spar break at 30\% of the semi-span (inset of Fig. 11). 
Local pockets of steering curvature will predominately impact the local metrics at that location (stress concentrations, panel buckling). Straight fibers that are rotated outside the pre-determined set (i.e., the $0^{\circ}$ fibers are no longer parallel to the wing leading edge) will impact the global bend-twist deformation characteristics of the wing, which will then have a downstream effect on stress and buckling throughout. Global changes in aeroelastic physics are further demonstrated in Fig. 12, which shows the sectional lift (ratio of flexible and rigid values) across the three aeroelastic load cases. Tow steering affords outboard load alleviation relative to the un-steered case (necessarily counteracted by higher lift inboard to maintain trim, which is clearly seen for the $2.5 \mathrm{~g}$ case), and augments the aerodynamic impact of the aileron during the roll maneuver.

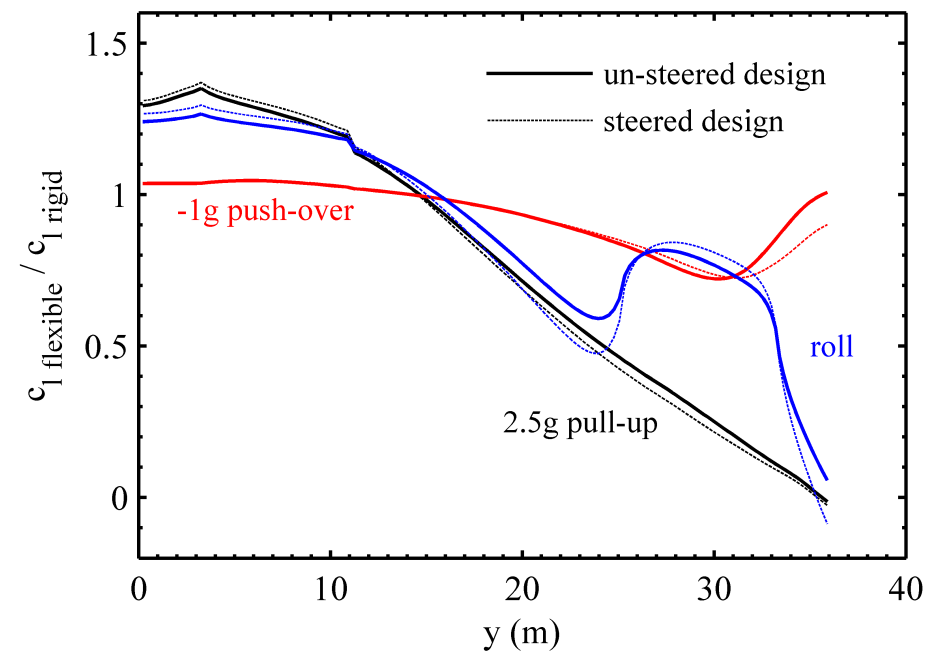

Figure 12. Ratio of the flexible and rigid sectional lift across three aeroelastic load cases.

\section{B. Synergies Between Tow Steered Composites and Curvilinear Stiffeners}

The final portion of this paper compares the benefits of curvilinear fiber paths and skin stiffeners, particularly when optimized in tandem. Two additional designs are presented here. Design 5 is a straight fiber wingbox with curved stiffeners (using the un-steered design 3 with straight stiffeners in Fig. 10 as a starting point), and design 6 has both curved fibers and curved stiffeners. Design 6 could reasonably use designs 3, 4, or 5 as starting points for the optimizer; all three options were tested, with minimal differences in the final converged result. All four composite designs are shown in Fig. 13 in terms of equivalent thickness distributions, and Fig. 14 in terms of optimal curvilinear paths along the skins.

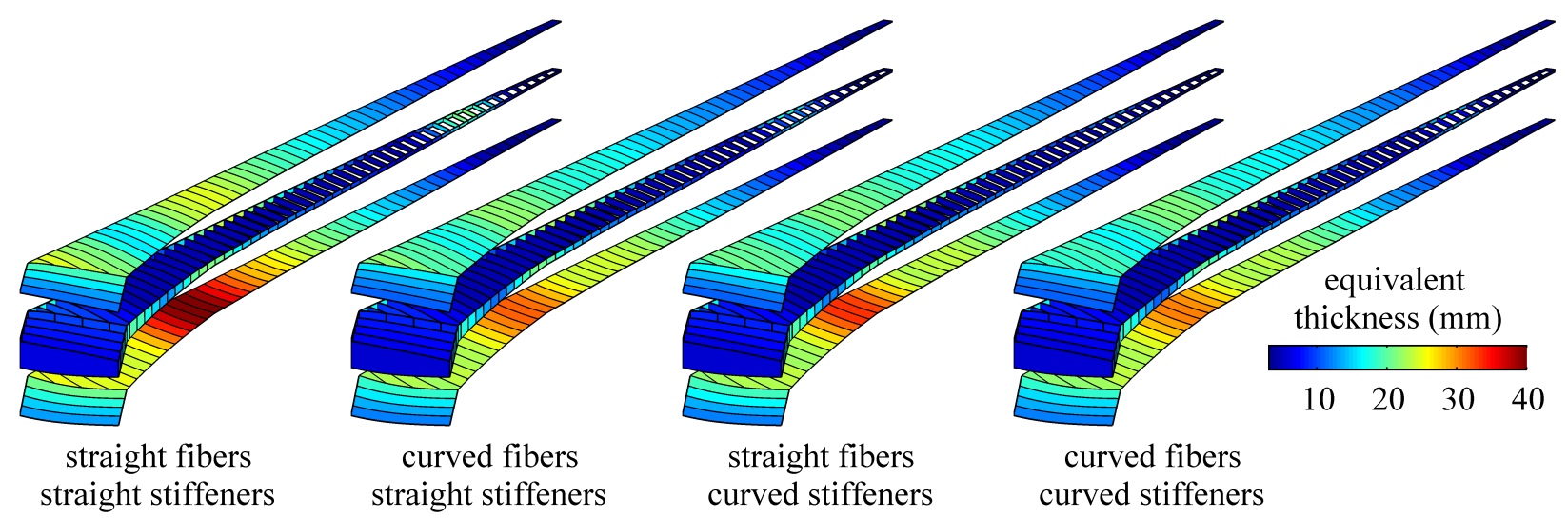

Figure 13. Equivalent wingbox thickness for composite designs with and without tow steering and/or curvilinear stiffeners (designs 3, 4, 5, and 6).

The optimal composite design with curvilinear stiffeners (but straight fibers) has an optimal mass of $13,751 \mathrm{~kg}$, an $8.5 \%$ reduction over the straight stiffener composite design. This is a comparable reduction 
to that achieved by design 4 , which reduced mass $8.8 \%$ via tow steering. The curved stiffener design has notably higher skin thicknesses in Fig. 13, but mostly compensates for this by removing the outboard web mass (used to mitigate flutter), a trade-off also noted for the metallic designs. It is perhaps surprising that the optimizer can use curvilinear stiffeners to obtain comparable mass reductions to tow steering: a curved stiffener is heavier than a straight one, while tow steering incurs no mass penalty relative to straightfiber designs (at least as envisioned here: it is possible to allow ply build-ups between adjacent curvilinear courses $^{8}$ ). On the other hand, skin buckling is strongly impacted by the larger moments of inertia afforded by curved stiffeners, as compared to skin ply tailoring.

The optimal mass with both tow steering and curvilinear stiffener design variables included (design 6) is $13,323 \mathrm{~kg}$, an $11.4 \%$ drop over the straight composite design. Including both sets of curvilinear design variables simultaneously provides a superior design to the case when either is considered alone, but the result is less than a true positive-synergy. These diminished returns are expected given that curvilinear stiffeners and tow paths use some (but not all) of the same techniques to improve aeroelastic performance. These techniques can be studied more closely in Fig. 14, where the results for the tow steered design (with straight stiffeners) is repeated from Fig. 11, albeit in a different graphical form.

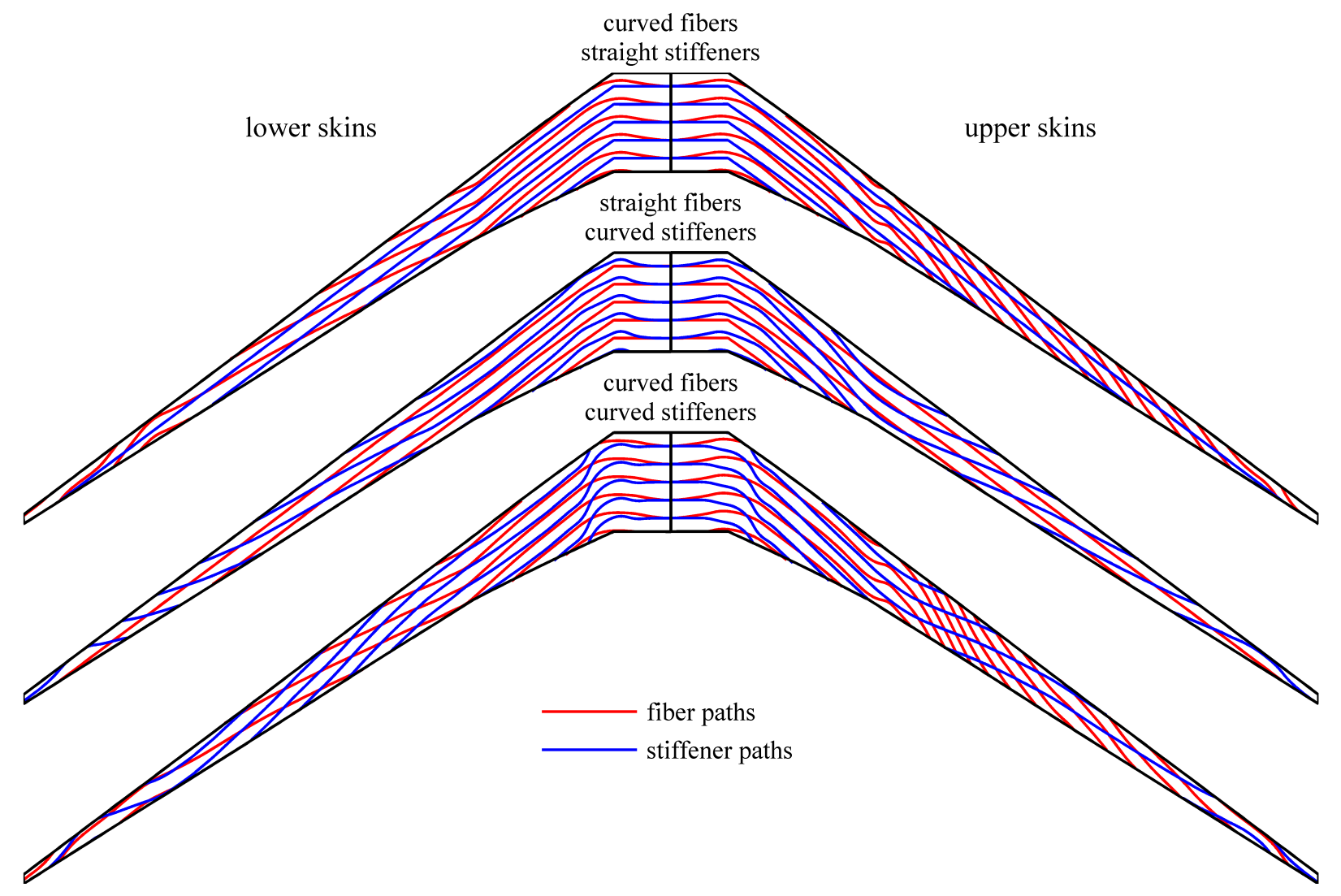

Figure 14. Curvature paths for composite designs with and without tow steering and/or curvilinear stiffeners (designs 4, 5, and 6).

As with the metallic cases, high localized stiffener curvature is seen at the wing root (due to the spar break) and tip (due to the local aileron loads), and more gradual curvature is also observed, spanning across several panels (particularly in the upper skins). This latter detail is in contrast to the optimal tow steering which, as noted, utilizes curvature mostly at a local level, and otherwise simply reorients the laminates to fiber directions beyond the prescribed set of four angles. Local tow steering is most notable at the two spar breaks in the wing geometry.

All of these trends largely hold when moving from individual technology optimization (designs 4 and 5) to simultaneous optimization (design 6), with the exception of more aggressive stiffener curvature at the wing root, and a switched sign of the stiffener curvature deviation angles at mid-span of the lower skins. This latter trend is interesting in that, if the optimizer had simply combined the steering patterns of design 
4 and the curved stiffeners of design 5, then for the lower skin of design 6, the two (fibers and stiffeners) would nearly align. Instead, the optimizer reorients the stiffeners so as not to be parallel to the fibers. It should be noted, however, that only the path of the $0^{\circ}$ fibers is shown in these figures, and the optimizer may be orienting the stiffeners to align closer to the $45^{\circ}$ fibers (seen in the upper skin of design 6 ) or the $-45^{\circ}$ fibers (lower skin). However, $\pm 45^{\circ}$ fibers are only predominant in the upper skins, a result not explicitly shown here, but qualitatively very similar to that shown in Fig. 10.

\section{Conclusion}

A summary of the six design cases obtained in this paper is given in Table 3. Key findings are as follows:

- All wingbox designs, in general, are strongly driven by aeroelastic stress and skin buckling constraints (with the stresses generated during the $-1 g$ maneuver the only set of metrics not approaching the failure boundary), and flutter constraints as well. The optimizer is forced to add lumped web mass towards the wing tip to quench the flutter instabilities.

- Adding curvilinear stiffeners to the metallic wingbox affords a $5.2 \%$ mass reduction, and the optimizer is able to remove the lumped web mass without violating flutter constraints. High local curvature is noted at the side-of-body and the aileron attachment locations, but gradual stiffener curvature spanning multiple panels is noted as well.

- Replacing the metal panels for composite panels represents a substantial increase in the size of the design space with a concomitant increase in the number of constraints. The optimal mass decreases by $6.9 \%$ relative to the metallic result, with asymmetrically higher thickness in the lower skins than the upper. The upper skins contain many laminates dominated by a single fiber direction, whereas fibers are more evenly distributed in the lower skins.

- Tow steering provides an $8.8 \%$ mass reduction relative to the un-steered composite wing. High local steering curvature is noted at the two breaks in the trailing spar, though large portions of the skin fibers are rigidly rotated from the pre-defined set of $0^{\circ} / 45^{\circ} /-45^{\circ} / 90^{\circ}$, without actually utilizing curvature.

- Adding curved stiffeners to the composite wingbox (with straight fibers) is more successful than when they are added to the metallic wingbox (8.5\% vs. $5.2 \%$ ), and only moderately less advantageous compared to tow steering.

- Utilizing both curvilinear stiffeners and tow steering provides further mass reductions (11.4\%) but overall diminished returns relative to the benefits of utilizing these technologies independently. This may be because the two techniques rely on several similar design strategies, though spatial trends in curvature bear many similarities between optimizing tow steering or curvilinear stiffeners separately versus optimizing them simultaneously, suggesting enough of a difference in the aeroelastic mechanisms exploited by the two schemes. Beyond the carry-through portion of the wing, the fibers and stiffeners prefer not to be parallel, despite the optimal fiber path in the straight-stiffener design, and the optimal stiffener path in the un-steered design, being oriented along similar paths for portions of the lower skins of those two wingboxes.

Table 3. Final design summary.

\begin{tabular}{rrrr} 
Design & Description & Optimal Mass & \# of DVs \\
\hline 1 & metallic wing: straight stiffeners & $16,146 \mathrm{~kg}$ & 849 \\
2 & metallic wing: curved stiffeners & $15,308 \mathrm{~kg}$ & 957 \\
3 & composite wing: straight stiffeners and straight fibers & $15,036 \mathrm{~kg}$ & 1305 \\
4 & composite wing: straight stiffeners and curved fibers & $13,700 \mathrm{~kg}$ & 1413 \\
5 & composite wing: curved stiffeners and straight fibers & $13,751 \mathrm{~kg}$ & 1413 \\
6 & composite wing: curved stiffeners and curved fibers & $13,323 \mathrm{~kg}$ & 1521
\end{tabular}

\section{Acknowledgments}

This work is funded by NASA's Advanced Air Transport Technologies program. Thanks to Dr. Joaquim Martins and Dr. Gaetan Kenway of the University of Michigan for providing the CRM finite element model. 


\section{References}

${ }^{1}$ Shrik, M., Hertz, T., Weisshaar, T., "Aeroelastic Tailoring - Theory, Practice, and Promise," Journal of Aircraft, Vol. 23, No. 1, pp. 6-18, 1986.

${ }^{2}$ Lukaszewicz, D., Ward, C., Potter, K., "The Engineering Aspects of Automated Prepreg Layup: History, Present and Future," Composites: Part B, Vol. 43, pp. 997-1009, 2012.

${ }^{3}$ Stanford, B., Jutte, C., Wu, K., "Aeroelastic Benefits of Tow Steering for Composite Plates," Composite Structures, Vol. 118, pp. 416-422, 2014.

${ }^{4}$ Stanford, B., Jutte, C., Wieseman, C., "Trim and Structural Optimization of Subsonic Transport Wings Using Nonconventional Aeroelastic Tailoring," AIAA Journal, Vol. 54, No. 1, pp. 293-309, 2016.

${ }^{5}$ Stodieck, O., Cooper, J., Weaver, P., Kealy, P., "Optimization of Tow-Steered Composite Wing Laminates for Aeroelastic Tailoring," AIAA Journal, Vol. 53, No. 8, pp. 2203-2215, 2015.

${ }^{6}$ Brooks, T., Martins, J., Kennedy, G., "High-Fidelity Aerostructural Optimization of a High Aspect Ratio Tow-Steered Composte Wing," AIAA SciTech Conference, San Diego, CA, January 4-8, 2016.

${ }^{7}$ Weaver, P., Potter, K., Hazra, K., Saverymuthapulle, M., Hawthorne, M., "Buckling of Variable Angle Tow Plates: from Concept to Experiment," AIAA Structures, Structural Dynamics, and Materials Conference, Palm Springs, CA, May 4-7, 2009.

${ }^{8} \mathrm{Wu}, \mathrm{K}$. , Turpin, J., Stanford, B., Martin, R., "Structural Performance of Advanced Composite Tow-Steered Shells with Cutouts," AIAA SciTech Conference, National Harbor, MD, January 13-17, 2014.

${ }^{9}$ Taminger, K., Hafley, R., "Electron Beam Freeform Fabrication: A Rapid Metal Deposition Process," Proceedings of the 3rd Annual Automotive Composites Conference, Troy, MI, September 9-10, 2003.

${ }^{10}$ Bhatia, M., Kapania, R., Evans, D., "Comparative Study on Optimal Stiffener Placement for Curvilinearly Stiffened Panels," Journal of Aircraft, Vol. 48, No. 1, pp. 77-91, 2011.

${ }^{11}$ Mulani, S., Slemp, W., Kapania, R., "EBF3PanelOpt: An Optimization Framework for Curvilinear Blade-Stiffened Panels," Thin-Walled Structures, Vol. 63, pp. 13-26, 2013.

${ }^{12}$ Stroud, W., Agranoff, N., "Minimum-Mass Design of Filamentary Composite Panels under Combined Loads: Design Procedure Based on Simplified Buckling Equations," NASA TN D-8257, 1976.

${ }^{13}$ Vassberg, J., DeHaan, M., Rivers, S., Wahls, R., "Development of a Common Research Model for Applied CFD Validation Studies," AIAA Applied Aerodynamics Conference, Honolulu, Hawaii, August 10-13, 2008.

${ }^{14}$ Kenway, G., Martins, J., Kennedy, G., "Aerostructural Optimization of the Common Research Model Configuration," AIAA Aviation Conference, Atlanta, GA, June 16-20, 2014.

${ }^{15}$ Cook, R., Malkus, D., Plesha, M., Witt, R., Concepts and Applications of Finite Element Analysis, Wiley, New York, 2002.

${ }^{16}$ Katz, J., Plotkin, A., Low-Speed Aerodynamics, Cambridge University Press, Cambridge, UK, 2001.

${ }^{17}$ Appa, K., "Finite-Surface Spline," Journal of Aircraft, Vol. 26, No. 5, pp. 495-496, 1989.

${ }^{18}$ Kreisselmeier, G., Steinhauser, R., "Systematic Control Design by Optimizing a Vector Performance Index", International Federation of Active Controls Symposium on Computer-Aided Design of Control Systems, Zurich, Switzerland, 1979.

${ }^{19}$ Dillinger, J., Klimmek, T., Abdalla, M., Gürdal, Z., "Stiffness Optimization of Composite Wings with Aeroelastic Constraints," Journal of Aircraft, Vol. 50, No. 4, pp. 1159-1168, 2013.

${ }^{20}$ Hassig, H., "An Approximate True Damping Solution of the Flutter Equation by Determinant Iteration," Journal of Aircraft, Vol. 8, No. 11, pp. 885-889, 1971.

${ }^{21}$ Zhang, Z., Yang, S., Chen, P., "Linearized Euler Solver for Rapid Frequency-Domain Aeroelastic Analysis," Journal of Aircraft, Vol. 49, No. 3, pp. 922-932, 2012.

${ }^{22}$ Chen, P., Gao, X., Tang, L., "Overset Field-Panel Method for Unsteady Transonic Aerodynamic Influence Coefficient Matrix Generation," AIAA Journal, Vol. 42, No. 9, pp. 1775-1787, 2004.

${ }^{23}$ Ringertz, U., "On Structural Optimization with Aeroelasticity Constraints," Structural Optimization, Vol. 8, pp. 16-23, 1994.

${ }^{24}$ Adelman, H., Haftka, R., "Sensitivity Analysis of Discrete Structural Systems," AIAA Journal, Vol. 24, No. 5, pp. 823-832, 1986.

${ }^{25}$ Bindolino, G., Mantegazza, P., "Aeroelastic Derivatives as a Sensitivity Analysis of Nonlinear Equations," AIAA Journal, Vol. 25, No. 8, pp. 1145-1146, 1987.

${ }^{26}$ Sigmund, O., "Morphology-Based Black and White Filters for Topology Optimization," Structural and Multidisciplinary Optimization, Vol. 33, pp. 401-424, 2007.

${ }^{27}$ Svanberg, K., "A Class of Globally Convergent Optimization Methods Based on Conservative Convex Separable Approximations," SIAM Journal of Optimization, Vol. 12, pp. 555-573, 2002.

${ }^{28}$ Alhajahmad, A., Abdalla, M., Gürdal, Z., "Optimal Design of Tow-Placed Fuselage Panels for Maximum Strength with Buckling Considerations," Journal of Aircraft, Vol. 47, No. 3, pp. 775-782, 2010.

${ }^{29}$ Niu, M., Airframe Structural Design, Conmilit Press Ltd., Hong Kong, 1988.

${ }^{30}$ Liu, B., Haftka, R., "Single-Level Composite Wing Optimization Based on Flexural Lamination Parameters," Structural and Multidisciplinary Optimization, Vol. 26, pp. 111-120, 2004. 